\title{
Textual Construction of Middle School Math Students as “Thinkers”
}

\author{
ALAYNE ARMSTRONG \\ University of British Columbia
}

\begin{abstract}
This paper investigates how the mathematical performance of a group of middle school students might be characterized when the text breaks from tradition and constructs students as members of the mathematical community. Firstly, I will consider how a current Canadian textbook presents The Locker Problem through a depersonalized, formalized style that promotes its authority over the student-reader (Rotman, 2006). Next I will argue that the presentation of the problem through a Problem-of-the-Week (POW) format promotes the author/ity (Povey, Burton, Angier \& Boylan, 1999) of the studentreader over the text. Finally, I will present a classroom episode where a small group of students explore the Locker Problem based on the POW format. While some have argued that one can infer the experience of the student-reader through a text's choice of words (Herbel-Eisenmann \& Wagner, 2007), I suggest that the student-reader's style of performing mathematics might also be inferred based on the text's presentation of a problem.
\end{abstract}

Ours is not to reason why

Just invert and multiply

\section{- Unknown}

Ask most North Americans about their experiences in school mathematics classes, and chances are that they remember opening the textbook to a certain page and following the directions listed there with lockstep precision. Perhaps for this reason, mathematics has gained a reputation as being a series of rules imposed by an outside source, be it textbook or teacher, with little recognition that student thinking might itself generate mathematics. In this paper, I follow up on a question posed by Herbel-Eisenmann and Wagner, wondering "how students' experiences of mathematics would differ if their textbooks did more to recognize persons, their contexts and mathematisation" (2007, p. 13). My study investigates how the mathematical performance of a group of middle school students might be characterized when the text breaks from tradition and constructs students as members of the mathematical community.

In this paper, I will explore how two middle school level texts present of "The Locker Problem." A "classic" problem that over the years has been adapted for use with students ranging from middle school age to university level, the Locker Problem can be found in many math resources and textbooks in many formats. In this problem, there is a long hallway lined with a number of lockers, and there is an equal number of students standing by. The first student comes along and opens all the doors to the lockers. A 
second student comes along and closes the doors to every second locker. A third student comes along and changes the door position of every third locker - if the door is open, she closes it; if the door is closed, she opens it. A fourth student comes along and changes the position of every fourth locker door, and so it goes. The problem asks, by the time the last student walks along and changes the position of the last locker door, which locker doors are left open?

Firstly, I will consider how a current Canadian textbook presents the problem through a depersonalized, formalized style that promotes its authority over the studentreader. Next I will discuss how the presentation of the problem through a Problem-of-theWeek (POW) format, based on the classroom work of Gary Tsuruda (1994), promotes the author/ity (Povey, Burton, Angier \& Boylan, 1999 of the student-reader over the text. Finally, I will discuss a classroom episode where a small group of students explore the Locker Problem based on the POW format. While some have argued that one can infer the experience of the student-reader through a text's choice of words (Herbel-Eisenmann \& Wagner, 2007), I suggest that the student-reader's style of performing mathematics might also be inferred based on the text's presentation of a problem.

\section{Textbook as Authority}

The first version of the Locker Problem I will discuss is found in a grade 8 textbook from the Canadian Math Makes Sense series (Baron et al, 2008). Rotman writes, "The only things mathematicians can be supposed to do with any certainty are scribble and think" (2006, p. 105), suggesting that their actions are some amalgamation of these two verbs. In their consideration of mathematics textbooks, Herbel-Eisenmann and Wagner further press this dichotomy, suggesting that "The 'thinker' imperatives construct a reader whose actions are included in a community of people doing mathematics, whereas the "scribbler" imperatives construct one whose actions can be excluded from such a community" (2007, p. 12). In the case of this particular presentation of the Locker Problem, the student-reader is a scribbler, merely following the dictates of the authoritative textbook.

The Locker Problem appears at the end of the first unit of the textbook, entitled "Square roots and the Pythagorean theorem," and is laid out on a two page spread designating it as the Unit Problem. Its location following the Practice Test section suggests that the Unit Problem is meant to play a role in reviewing the content of the unit.

The problem is described in a very direct manner, with an obvious effort to spell out what happens as each of the first, second, and third students proceed down the row of lockers. It is notable that the problem takes the simplest form possible while still providing a large enough quantity of lockers (25) to work with in order for a pattern to appear. The question itself is stated at the very end of the description of the situation: which lockers will be open after the $25^{\text {th }}$ student has passed down the row?

The student-reader is then given a series of questions that will direct her step-bystep through a solution for the problem. Question 1 presents these directions through a set of exclusive imperatives - imperatives which require obedience but little in the way of thought on the part of the student-reader (Rotman, 2006). The student-reader is first told to "Draw a chart similar to the one below" but to "Extend the chart" to include all 25 lockers and is even told what symbols to use within the chart "Write $\mathrm{O}$ for each opened locker;" "Write C for each locker that was closed;" "Write C or O for each locker that 
was changed"). The stub of a chart is provided, the data for the first three students are already filled in, and the student-reader is instructed to "Complete the chart for 25 students" (Baron et al., 2008, p. 60).

Once the chart is completed, the student-reader is expected to move on to Questions 2-5. First, the question from the original situation is repeated almost word-forword. The student-reader is then asked "What pattern do you notice?" the assumption being that there is a pattern to notice and that the student-reader will notice it. This is followed by "How can you use this pattern to tell which lockers will be open?" where, once again, the assumption is that this pattern can be used.

With the idea of looking for a pattern presumably planted, Question 3 seeks to extend the situation. The student-reader is directed to "Suppose there were 100 lockers and 100 students" and asked which lockers would remain open. Question 4 makes a further extension - suppose there are 400 lockers and 400 students. Finally, Question 5 directs the student-reader to use the pattern to make a rule - What is the rule for any number of lockers and students? - and then asks for an explanation - Why do you think your rule works?" Again the assumption is that there is a rule, the student-reader will be able to find it, will think that it works, and be able to explain it.

If word problems are a genre (Gerofsky, 2004), then the presentation of the Locker Problem in this text could be considered to belong to a special kind of genre, one that offers a specific set of instructions leading to a specific result - the recipe. In a recipe, the answer is predetermined. In fact, it's usually the title of the text: for example, the heading "Oatmeal Cookies" implies that if you follow all the steps correctly, your final result (answer) will be oatmeal cookies. In this instance of the Locker Problem, the answer is revealed by the location of the problem (at the end of a unit about square numbers) and by broad hints given by the instructions for Part B of the problem: "Copy and complete a chart similar to the one below for the square numbers to 900" (Baron et al, 2008, p. 61). If it has not yet occurred to the student-reader that all of the open lockers have square numbers, it probably will now.

In Part B of the Unit Problem, the original focus of the Locker Problem itself changes to force an extension of the problem, namely a search for Pythagorean triples. Again, a stub of the chart is provided with the first few rows already completed. The language briefly becomes inclusive as the purpose of the chart is introduced - "We can use the completed chart to find some Pythagorean triples" - and an example of a Pythagorean triple is provided. At this point, the text offers some leading questions, seeking to point the student-reader towards patterns contained within the chart in order to identify more Pythagorean triples. However, there is no explanation as to why the concept of triples is even being raised, nor the student-reader encouraged to consider why it might be important. The student-reader is simply expected to follow the instructions.

This abrupt turn in the direction of the Unit Problem appears to have been contrived in order to review the contents of the unit itself; the Pythagorean Triples were first introduced 20 pages earlier in the textbook, although there is no direct reference to these pages in the Unit Problem. This review focus is reinforced at the end of the Unit Problem's two page spread with a section entitled "Reflect on Your Learning." This refers not to the Locker Problem itself, nor to the Pythagorean triples extension, but to the application of the Pythagorean theory itself, again something which appeared earlier in the chapter. In this textbook, the Locker Problem has been introduced and then discarded 
in order to fit the outcomes of the unit: namely, to cover the concepts of square numbers and Pythagorean theory.

\section{Problem of the Week as Author/ity}

Mathematics is ever evolving. Lakatos, the philosopher who first set this idea out clearly (Ernest, 1998), depicts mathematics as a process, an aspect of culture that develops through never-ending discussion and argument - there is always something else to consider (Lakatos, 1976). School mathematics, however, has traditionally treated mathematics as a set of static ideas to be transmitted to students by their texts and teachers. For students, "knowing" mathematics is often to be aware only of the rules and theories themselves, and not the history and discussion, the human element, behind them and, as a result, mathematics becomes "a cultural form suffused with mystery and power" (Povey et al, 1999, p. 235). Textbooks, ever present in the classroom, take on the authority to wield that power, shaping student notions of mathematics in the process (Love \& Pimm, 1996).

Povey et al (1999) use the term author/ity to play with the concept of authority the traditional view of mathematical knowledge as external, fixed and absolute - splitting up the word to foreground the author behind the scenes, the one who negotiates and creates this knowledge. In this scenario, they write, "teachers and learners... work implicitly (and, perhaps, explicitly) with an understanding that they are members of a knowledge-making community.... As such, meaning is understood as negotiated. External sources are consulted and respected, but they are also evaluated critically by the knowledge makers, those making meaning of mathematics in the classroom with whom author/ity rests" (Povey et al, 1999, p. 234). A knowledge-making community counters the role of textbook as the authority in the classroom. Responsibility passes to the students to break away from playing the role of empty vessels waiting to be filled with facts and formulae, and instead to make meaning of mathematics for themselves.

In contrast to the previous section, where the problem was found within a textbook, the POW format presents the Locker Problem to student-readers on an individual sheet of paper (Appendix A). This problem is not embedded in a specific unit of study, and thus it does not have a specific location in regards to other mathematical texts. Its underlying purpose, then, is not set by its location but only by the educator who chooses when, where and why to assign the problem. On its own, the problem's purpose is to provide a puzzle that is an exploration of number theory.

The generic introduction at the beginning of the problem (Problem Statement, Plan, Work, Answer) is the same as for all POWs used throughout the school year in the particular classroom that took part in this study. There are no leading questions specifically in place for this problem. There is no embedded answer. In fact, these instructions are quite subversive. Although the POW text reserves the authority to ask questions of the student-reader, it does not reserve the authority to determine "the" solution. That it leaves to the student-reader. In fact, it encourages the student-reader to present an account that is fully grounded in her own experiences, past, present and future, both as a mathematician and as an author.

The instructions open with an imperative for the student-reader to write a concise statement, but qualifies this - the student-reader is asked to write clearly enough for any potential reader. In a sense, the student-reader is immediately set up as an author with this 
suggestion of future readers. Next is the Plan. Here the imperatives are all geared towards the student-reader's experience. How did the problem seem to you? Consider what you are asked to find (a reference to the problem itself but one that is related back to the student-reader). There are references to the student-reader's background (What do you know), needs (What do you need to know? - a suggestion that resources and ideas will need to be gathered), and connections (the student-reader's experience with other problems). The student-reader is asked to make a guess, but there is no suggestion as to what a "correct" guess would be. The imperatives in this section are inclusive - the student-reader is invited to think and then respond (Rotman, 2006).

In the Work section, there are no prescribed step-by-step instructions to follow. Instead, the student-reader is directed to report her own experience in detail, and to use charts and graphs where she deems it to be appropriate (i.e., she is asked to use her own judgment). What's interesting here is that not only is student-reader asked to describe what worked, but to also describe what did not work and where she got stuck. This approach recognizes that errors and cul-de-sacs are as valid a part of the mathematical experience as getting the "right" answer is. There is no pressure to provide an official (and false) narrative that outlines a smooth and inevitable path to a correct solution. Also working against the official narrative is the suggestion that the student-reader may use the community around her for assistance - Did you get help from anyone? What kind of help? Gone is the image of the isolated, independent mathematics student. Instead there is a recognition that mathematics students work with others.

Finally, even the idea of the answer as marking the end of the process is disrupted. The student-reader is asked to state the answer, but then is immediately asked if it makes sense and if there could be alternate solutions. In short, the student-reader is asked to provide a judgment as to the quality of her proposed answer. There are references to the past (Compare your answer with your original guess) as well as the future (What did you learn from this that might help you solve other problems?).

So while there are imperatives present in the POW text, they are directions that solicit the student-reader's experience rather than her acquiescence in performing certain actions. These imperatives are interspersed with questions that are actual questions, not "questions" which serve to lead the student-reader to perform certain tasks. An alternative mathematical reality is posited - not an individual formalized frog march to a predetermined "right" answer, but one where bricolage is the promoted practice. The term bricolage originates from the French verb bricoler - to tinker, to fiddle with, or, in contemporary French, to "do it yourself" with the materials available to you - and some consider it to be "a style of organizing work that invites descriptions such as negotiational rather than planned in advance" (Turkle \& Papert, 1990, p. 144). The POW instructions suggest that the student-reader should work with what she has - her experiences, her resources, and those of the community around her - and that it is natural to get stuck, to share ideas, to arrive at alternate solutions, to test one's ideas against others' to check their soundness. And finally, the implication is that it is all part of an ongoing process one will always be gathering ideas and resources that may prove useful in the future.

In summary, what the introductory text of the POW does is disrupt its own potential authority and instead offers author/ity to the student-reader with nudges/instructions to provide detail, clarity and reasoning rather than mandating a particular direction or method. This, I argue, provides fertile ground for student-readers to 
act as bricoleurs in approaching a mathematics problem. I will now discuss an episode from a classroom where small groups of students were assigned the Locker Problem presented in the POW format.

\section{Method and Data Sources}

This paper focuses on a group of students (Anik, Crystal, Khona and Sandi) who are working on the Locker Problem, and is an excerpt from a larger study exploring the characteristics of group flow (Author, 2005). The research took place at a middle school in a large suburban school district in British Columbia in the spring of the school year. The class videotaped was one of my own Grade 8 mathematics classes, selected because the class was relatively homogeneous in mathematics ability and group work was part of the classroom culture. The sampling was purposeful: subjects were selected based on their willingness to participate in the study, their ability to express themselves, and their comfort with collaborative work. There were two video cameras each recording the activities of a group (which varied in size from four to six members, depending on the session) in the classroom for each 40 minute session, for a total of four sessions. The POW question was a task with routines that were familiar to the class, and was chosen to provide opportunities for collaboration. During the course of the school year, students had come to understand that they were to work on POWs as independently from the teacher as possible - i.e., that I, as teacher, would clarify information in the problem if they asked, but would provide no hints about what to do, and no suggestions as to whether a proposed answer might be right or wrong. This established routine meant that students expected that I, as teacher/researcher, would leave the groups alone (barring any behavioral issues), whether or not a group was being videotaped.

All data gathered through video and audio technology and from participant documents and artifacts were collected with explicit permission from the participants and their parents and in full compliance with the Behavioral Research Ethics Board guidelines of my university. Permission for the project was granted by the school's administration. Students in the class were informed about the research and were provided with assent forms for themselves and consent forms for their parents/guardians by a teacher colleague who managed the distribution and collection of these forms. Those students who chose not to participate in the study still took part in the mathematical tasks, but the groups they worked in were not videotaped or audiotaped, and I did not formally observe them for my research. Other than doing a quick check to see if the video and audio recorders were recording, I did not view or listen to the recordings until the school year had been completed.

Although both boys and girls took part in the larger study, this article focuses on a group who was made up of girls. I made this choice based on the clarity and enthusiasm of this particular group's discussion about the math problem.

The data were analyzed using constant comparison method (Glaser \& Strauss, 1967). After the transcription was completed (itself a layered and iterative process), I started categorizing by reading and rereading the transcript to identify problems posed by the group in its discussion. Initially this was done intuitively, but through a constant comparison, eventually categories of problems posed began to emerge and these were recorded. 


\section{The Study}

This section will focus on excerpts from the discussion of an engaged group of four girls - Sandi, Khona, Anik and Crystal - as they work their way towards a solution. The group begins by working with what they had, namely, the information provided by the problem. First they focus on which students changed the door positions of which particular lockers.

[9] Anik: $\quad$ First student opens all of them

[10] Sandi: Yeah, so he opened all of them

[11] Unknown: Second student

[12] Unknown: I think I got it.

[13] Unknown: Closed all of the uneven ones

[14] Sandi: $\quad$ All of the even ones

[15] Khona: Uneven ones

[16] Unknown: No he closed the even ones

[17] Sandi: Then the third one,

[18] Khona: $\quad$ Oh

[19] Sandi: um, numbers 3, 6, 9, 12, you know up like that, if they were open he'd close them, and if it were closed he'd open it, whatever those numbers are

[20] Anik: Like the 3?

[21] Sandi: $\quad$ Yeah... multiples of 3

[22] Sandi: Yeah. And then fourth student multiples of 4 change the position to either...

[23] Anik: $\quad$ And then there'd be the multiples of 5

[24] Sandi: Yeah

[25] Anik: 6

[26] Sandi: And the multiples of every other number, up to 1000

In their subsequent discussion, a series of mathematical questions emerge, which I have paraphrased as follows:

- What if we consider multiples?

- What's the overall pattern needed to solve this problem?

- What if it's a trick question?

- What if a fractional proportion of the lockers are opened?

- What about numbers with only two factors (prime numbers)?

- How many prime numbers are there between 1 and 1000 ?

- What is the pattern of prime numbers between 1 and 100 ?

- What is the pattern of prime numbers within each block of 10 numbers (e.g. 1-10; $11-20$, etc.)?

- What if the pattern has to do with the number of factors in each number?

- Which numbers have an odd number of factors?

Note how the central problem under consideration changes as the group members proposed various questions and tinker with the ideas that develop. By the end of the 
session, the girls have thoroughly considered the nature of multiples, factors, and prime numbers and are on the verge of identifying the pattern as being that of square numbers.

In working with the information provided in the problem, the group members also draw on their previous experiences in working on mathematics problems. They begin by considering "easy" strategies that have served them well in the past, ones that involve how to handle the information by the problem.

[37] Sandi: I always just start writing all the numbers out but there's a thousand here and I'm not going to write a thousand numbers.

[65] Anik: I can do the easy thing - highlighter, anyone have a highlighter? I'm going to highlight all the useful information.

Although they use the strategy of looking for a pattern early on (for instance, when working with multiples), no one states this strategy aloud until later in the session ([300] Anik: I think we have to look for a pattern).

The girls also refer to expectations about how to proceed with the problem, for instance, when the group is trying to determine the pattern of prime numbers within each block of 10 .

[332] Sandi: $\quad$ For numbers 1 to 10 there's 3 that stay open and 7 that stay closed, 11 to 20, 5 are open and 5 are closed

[333] Anik: How about number?

[334] Sandi: I'll do up to, okay, I'll just do up to 50

[335] Anik: Do you know what? I'll go up to, like,

[336] Sandi: $\quad$ Kay. So.

[337] Sandi: What do you want me to go up to? 30 or?

[338] Anik: (?)

[339] Sandi: $\quad$ Are you joking? (laughter) Oh my.

[pause]

[340] Anik: The answer would be a long list of numbers.

[341] Sandi: obviously she's not going to make us, like, write down a whole bunch of numbers, like it says

[342] Anik: What is the actual point of this?

[343] Sandi: It says how many, or, which doors are open

[344] Anik: Yes

[345] Sandi: $\quad$ So there's not that many.

[346] Anik: Yeah, I know but he's saying (?)

[347] Sandi: Yeah, I know but is he saying 600 doors are open or number 600 is open

[348] Anik: I don't know but I think more than one door would be open

[349] Sandi: Yeah

[350] Anik: $\quad$ But I think less than 600

[351] Sandi: Yeah

[352] Anik: Would be open

[353] Sandi: Because she's saying which doors she's not going to make us write down 600 doors. 'Kay I'll go up to 40 I guess. 
Although these references are mostly to what the teacher's expectations would be [341 \& 353], namely that there is no need to write down a lot of numbers in order to solve the problem, the girls also mention an answer of 600 that a boy from another group has recently called out [346]. The girls consider how reasonable 600 would be as an answer, conclude that it is too high a number, and decide to look at the first 40 lockers in order to find a pattern.

As the group works and tinkers with ideas, they also draw on external resources as needed. After quite a bit of time working together drawing charts, working with the idea of multiples, and looking for patterns, the following conversation emerges:

[120] Anik: Wait wait I know what we can do (?) we know (?) don't have any (?) Like 17, you can't times any number to get 17 (?) is that right?

[121] Sandi: Yeah

[122] Anik: $\quad$ So 17 would always be open. So we can use that to figure out which ones will always be open.

[123] Sandi: $\quad$ So where do we start?

[124] Anik: I'm not quite sure.

[pause]

[125] Sandi: $\quad$ So those numbers will stay closed

[126] Anik: $\quad$ No it stays open

[127] Sandi: $\quad$ So the first one opens all of them, and when it gets to their number they open that one so it'd be closed

[128] Khona: Prime numbers, are they called prime numbers?

[129] ?: $\quad$ Yeah the ones that can't

[130] Anik: Oh, I can't remember

At first the group's discussion centers on the name and properties of prime numbers, in particular that these numbers only have two factors each, but when the girls reach the limit of what they can remember, they turn to other resources.

[196] Khona: How many prime numbers are there?

[197] Crystal: I know, we have the sheet

The sheet Crystal refers to is from a class activity four months earlier which modeled the Sieve of Eratosthenes as a way of determining all the prime numbers between 1 and 100. (In this activity, each of the numbers in this range is systematically checked to see if other numbers can divide evenly into them. Those numbers which can only be divided by 1 and themselves - for example, 17 can only be divided by 1 and 17 are identified as prime numbers). The girls proceed to check their binders, a nearby group of students, and then their lockers, and once they finally locate a copy of the sheet, they use it as a basis for determining a pattern for seeing how many prime numbers there are between 1 and 1000. When there does not seem to be a visible pattern between 1 and 100, they decide to break the numbers up into groups of 10 [i.e., 1-10, $11-20$, etc.] to see if they might be able to locate any patterns that way. After the girls have worked awhile, Anik suggests how they might use the idea of factors in a different way. 
[370] Anik: Wait, I just figured something out, look.

[371] Sandi: What?

[372] Anik: If you figure out how many times any number... 1000 goes into each number, like, Say you pick the number 20. You know 2 can go in it, into it, 4 can go into it, 5 can go into it, 10 can go into it, and if I'm right that's it. So you know four numbers can go into it.

[373] Sandi: Right.

[374] Anik: It starts open, or it starts closed, so it will end closed. Or, I mean it will end open.

[375] Sandi: Oh my god, you're so smart Anik.

[376] Anik: Closed, open, closed, open

[377] Sandi: Yeah.

[378] Anik: It will end open. And if it's odd then it will end the same as it started. So it will be even if the number of numbers that goes into it is, I don't know if I'm making any sense

[379] Sandi: 'Kay you know what? You're smart.

Upon further discussion, the entire group accepts this new idea. Now wondering which numbers have an odd number of factors, the group draws on its resources again, and for the last few minutes of the session, the girls use a multiplication chart to help determine how many factors certain numbers have.

\section{Discussion}

Mathematicians have been characterized as being both "scribblers" and "thinkers" (Rotman, 2006), and in this paper I've explored how students might also inhabit this dual role. A textbook with its exclusive imperatives treats the student as a scribbler; the POW with its inclusive imperatives treats the student as a thinker. There is nothing wrong with providing guiding structures within which students can work, particularly for a new or difficult topic. The traditional textbook format is perpetuated in part because it seeks to help the individual student who may lack outside support in engaging with the ideas in the text (i.e. the text itself must act as teacher) (Love \& Pimm, 1996). However, when a textbook designed for classroom use privileges its own authority to the point where students are no longer expected to draw on their own resources - or, more bluntly, think about what they are doing- then the text is ultimately working against the process of learning.

Love and Pimm note that our society tends to believe that "text materials have always been the way they are today. This perspective is of particular importance in mathematics, because of the avowedly strong continuity of tradition of problem form (and sometimes actual problems), and of the context and situations offered in text materials" (1996, p. 373). Even a textbook series like Math Makes Sense which claims to have "A consistent three-part lesson model [that] supports problem based learning which helps students gain a deep understanding of mathematics and become lifelong problem solvers" 1 ends up bowing under the weight of traditional textbook step-by-step mechanisms. 
Herbel-Eisenman and Wagner suggest that, in a text, "The 'thinker' imperatives construct a student-reader whose actions are included in a community of people doing mathematics, whereas the 'scribbler' imperatives construct one whose actions can be excluded from such a community" (2007, p. 12). In offering author/ity to its readers, the POW offers an invitation for students to join the community. As the group works on the Locker Problem a number of questions emerge in their discussion. None of these questions are prescribed by an external source - not even by the POW instructions themselves. What the girls do is explore the nature of square numbers and their factors, wandering along a narrative path that evolves as they go.

Herbel-Eisenmann and Wagner characterize "mathematisation as the moves between the personal and impersonal, between context and abstraction. Mathematics lives in this tension" (2007, p.13). This movement, I suggest, is bricolage. The students work with what they have, both in mind (what they personally know and remember about mathematics that appears to relate to the problem, and their understanding of the sociomathematical norms associated problem solving) and on hand (external resources such as prime number and multiplication charts). As the girls discuss their ideas, they tinker with them, moving "between the personal and impersonal", developing and evolving the ideas as they go. By providing an open space for student-readers to draw on their own resources and processes, the wording of the POW instructions encourages the girls to join the mathematical community and ultimately to take a rich learning journey through the mathematics of the Locker Problem.

\section{References}

Author. (2005). Group flow in small groups of middle school mathematics students.

Unpublished master's thesis, University of British Columbia, Vancouver, British

Columbia, Canada.

Baron, L., Brown, T., Davis, G., Jeroski, S., Ludwig, S., Milne, E., Neel, K., Pusic, J., Sidley, R., \& Sufrin, D. (2008). Math Makes Sense. Toronto: Pearson Education Canada.

Ernest, P. (1998). Social Constructivism as a Philosophy of Mathematics. Albany, NY: State University of New York Press.

Gerofsky, S. (2004). A Man Left Albuquerque Heading East. Word Problems as Genre in Mathematics Education (Vol. 5). New York, NY: Peter Lang.

Glaser, B. G., \& Strauss, A. L. (1967). The Discovery of Grounded Theory: Strategies for Qualitative Research (1st ed.). Chicago: Aldine Publishing Company.

Herbel-Eisenmann, B., \& Wagner, D. (2007). A framework for uncovering the way a textbook may position the mathematics learner. For the Learning of Mathematics, 27(2), 8-14.

Lakatos, I. (1976). Proofs and refutations. The logic of mathematical discovery. Cambridge: Cambridge University Press.

Love, E., \& Pimm, D. (1996). 'This is so': a text on texts. In A. J. Bishop (Ed.), International Handbook of Mathematics Education (pp. 371-409). Netherlands: Kluwer Academic Publishers. 
Povey, H., Burton, L., Angier, C., \& Boylan, M. (1999). Learners as authors in the mathematics classroom. In L. Burton (Ed.), Learning Mathematics: From Hierarchies to Networks (pp. 232-245). London: Falmer Press.

Rotman, B. (2006). The semiotics of mathematics. In R. Hersh (Ed.), 18 Unconventional Essays on the Nature of Mathematics (pp. 97-127). New York: Spring Science+Business Media, Inc.

Tsuruda, G. (1994). Putting It Together. Portsmouth, NH: Heinemann.

Turkle, S., \& Papert, S. (1990). Epistemological pluralism: styles and voices within computer culture. Signs: Journal of Women in Culture and Study, 16(1), 128-157.

Author Biography

Dr. Alayne Armstrong is currently a lecturer/adjunct professor in the Department of Curriculum and Pedagogy at the University of British Columbia. 